\title{
Visual Discrimination Accuracy across Reaction Time in Rats
}

\author{
Daniel D. Kurylo*, Cindy Lin, and Taylan Ergun \\ Department of Psychology, Brooklyn College CUNY, Brooklyn, NY \\ *Corresponding author (Email: dkurylo@brooklyn.cuny.edu)
}

Citation - Kurylo, D. D., Lin, C., \& Ergun, T. (2020). Visual discrimination accuracy across reaction time in rats. Animal Behavior and Cognition, 7(1), 23-38. doi: https://doi.org/10.26451/abc.07.01.03.2020

\begin{abstract}
Processing time, computational load, and stimulus strength interact in perceptual decisions. Studies with non-human primates and rodents have demonstrated that perceptual decisions are guided by stimulus processing as well as cognitive factors, such as decision confidence. For both olfactory and visual discrimination, mice and rats demonstrate a trade-off between accuracy and speed, which is mediated by the level of difficulty and amount of sampling time. In order to investigate the relationship between performance and response time for a discrimination task in which rats controlled trial initiation, response frequency and accuracy were measured across reaction time (RT). Rats discriminated visual patterns consisting of solid-lines (low computational load) or dot grids (high load). Stimulus contrast and duration were varied across conditions. Results indicated a peak function across RT with two phases: an earlier component where accuracy improved to a peak level, followed by a later component where accuracy remained stable or declined. For the earlier phase, reduced salience slowed processing with higher computational load. Unexpectedly, peak performance with highly salient stimuli occurred more quickly with higher computational load. For the later phase, accuracy did not benefit from longer RT, but instead progressively declined. Pre-peak characteristics suggest that accuracy benefits from increased stimulus processing time and is consistent with computational models of progressive accumulation of stimulus information. Post-peak characteristics may reflect factors associated with perceptual decision, such as uncertainty, which delays committing to a response. Results thereby suggest separate effects of stimulus processing and perceptual decision on discrimination accuracy across RT.
\end{abstract}

Keywords - Animal behavioral assessment, Animal psychophysics, Perceptual grouping, Processing latency

Accurate evaluation of stimulus conditions enables animals to make appropriate perceptual decisions and effective behavioral responses. Such response selection is composed of a sequence of components, including stimulus processing, cognitive functions associated with decisions, and the programming and initiation of behaviors (Hare et al., 2011; Schall, 2001; Sternberg, 2011). Latencies and durations of processing components vary with stimulus quality and task demands (Sigman et al., 2007). In addition, a trade-off exists between response speed and accuracy, which varies across trial parameters (Abraham et al., 2004; Heitz, 2014; Heitz \& Schall, 2013; Mulder et al., 2013; Plainis \& Murray, 2000; Reinagel, 2013a,b; Reppert et al., 2018; Rinberg et al., 2006; Uchida \& Mainen, 2003). By systematically controlling available information, the relationship between accuracy and RT may be used to examine the time scales of underlying processes.

For a given set of task conditions, RT from stimulus onset can vary substantially. For example, for a visual discrimination task, responses occur across the first $600 \mathrm{~ms}$, and continue with reduced 
frequency to beyond one second (Cassey et al., 2014; Ratcliff \& Rouder, 1998; Reppert et al., 2018). Variability in RT may stem from many sources, including stimulus processing, choice certainty, and motoric factors. In addition, accuracy varies across RT, affected by the level of stimulus processing and the progress of perceptual decisions. The well-known trade-off between emphasizing speed versus accuracy is mediated by the level of information acquired, which varies with rate of acquisition as well as quality of the information (for a review, see Heitz, 2014). Examining discrimination of coherent motion in random dot arrays, it was reported that factors affecting accuracy across RT include processing efficiency and strength of neural representations, (Palmer et al., 2005), as well as mechanisms associated with perceptual decision (Kiani \& Shadlen, 2009). As such, increased percentage of coherent motion produced an increase in accuracy and a decrease in RT. Such factors are related to stimulus strength as well as computational load placed on stimulus processing. Both stimulus characteristics and task demands thereby affect accuracy across RT.

Increased RT enables additional processing time. Mathematical models of response time describe the continuous accumulation of stimulus information across time, and account for numerous experimental conditions (Ratcliff \& Rouder, 1998). Increased RT may also reflect a delay in response due to uncertainty, which produces hesitation in making a response and extends RT. Examining neural correlates of decision confidence in monkeys (Kiani \& Shadlen, 2009) and rats (Kepecs et al., 2008), increased neural response accompanied increased levels of confidence, providing physiological evidence linking confidence to choice decision.

The relationship between RT and accuracy has been studied extensively in humans and nonhuman primates at both a perceptual and physiological level (Heitz \& Schall, 2012, 2013; Roitman \& Shadlen, 2002). Less research has been conducted with rodents, despite their increasing use in perceptual studies (Carandini \& Churchland, 2013; Gavornik \& Bear, 2014; Zoccolan, 2015). To this end, efficient procedures have been developed for training and behavioral assessment in freely behaving rats (Kurylo et al., 2015; Reinagel 2013a, b; Soma et al., 2014; Tafazoli et al., 2012), facilitating analysis of perceptual capacities and physiological mechanisms.

In the olfactory domain, rats and mice are able to accurately discriminate odorants in as little as $200 \mathrm{~ms}$ from stimulus onset. Uchida and Mainen (2003) trained rats to nose poke a central odor port and discriminate odor pairs by withdrawing their head and moving to a left or right response port. Task difficulty was controlled by varying the similarity of the odor pairs. Discrimination accuracy was $97 \%$ correct for the most dissimilar odors and declined (to approximately 92\%) with increased odor similarity. Across difficulty level, median discrimination times were nearly identical at approximately $226 \mathrm{~ms}$. Tracking performance across RT, discrimination accuracy progressively increased across the first $200 \mathrm{~ms}$, then remained relatively stable across longer RT.

Using a go-no-go paradigm with mice, Abraham et al. (2004) found a similar time-scale for odor discrimination. However, the median discrimination time increased with increased similarity of odor pairs. Mice were trained to nose poke an odor port and remain within the port for one odor, for which they received reward, and no reward was provided for the alternate odor. Discrimination time was measured as the time at which response differences occurred between the odor pair relative to stimulus presentation. The median discrimination time for highly dissimilar odor pairs was $269 \mathrm{~ms}$, which increased to $490 \mathrm{~ms}$ for similar pairs. For each level of discrimination difficulty, mice maintained a high level of accuracy. These results provided evidence of speed-accuracy tradeoff under these experimental conditions.

Rinberg et al. (2006) directly tested the speed-accuracy tradeoff in mice by controlling sampling time. Mice were trained to nose poke a central odor port, and to discriminate odor pairs by moving to an adjacent reward port to the left or right. Discrimination difficulty again varied with the level of similarity between odor pairs. In addition, mice were trained to remain within the odor port until a buzzer sounded. Buzzer onset ranged from zero to one second from stimulus delivery, thereby varying the odor sampling time. For the enforced sampling conditions, accuracy improved with increased sampling time, demonstrating speed-accuracy tradeoff. For dissimilar odor pairs, accuracy peaked at approximately 300 $\mathrm{ms}$, whereas peak accuracy occurred at $600 \mathrm{~ms}$ for similar odors, indicating that the level of difficulty is a primary factor accounting for peak performance on the discrimination task. 
In the visual domain, the pattern of RT response frequency for a discrimination task occurred at a similar time scale as that found with odor discrimination (Reinagel, 2013b). Using a self-paced operant procedure, rats were trained to lick a central port, which immediately triggered the appearance of a visual stimulus on a CRT monitor. Rats were trained to discriminate coherent motion direction by moving to a left or right response port. Rats viewed displays of randomly positioned dots. A percentage of the dots moved in a common direction, whereas the remaining dots moved in a random direction. Thresholds represented the lowest level of motion coherence at which the global direction of dot patterns could be discriminated. Rats were allowed unlimited time to respond. It was found that discrimination accuracy progressively increased with increased RT. In addition, there was a steeper rise in accuracy with higher levels of motion coherence. These results are consistent with the progressive accumulation of stimulus information, where the rate of accumulation is related to stimulus quality.

As with a random dot motion task, discrimination accuracy increased across RT using static images that did not modulate across time (Reinagel, 2013a). Rats discriminated a pair of similarly shaped real-world objects presented together. Discrimination difficulty was varied by morphing image pairs. It was found that mean accuracy progressively improved with increases of RT up to $2 \mathrm{~s}$. In addition, accuracy decreased and RT increased with increased discrimination difficulty. Whereas the stimulus changed across time with studies employing the coherent motion condition (movement of noise elements and coherent motion elements), no change occurred with the static images. This change in accuracy across RT therefore is not attributed to within-trial changes intrinsic to stimuli, but instead reflect aspects of processing, such as sensory processing or factors associated with discrimination and decision.

The relationship between response latency and discrimination accuracy is not clear. Increased stimulus quality improves accuracy, which is consistent with increased discrimination accuracy across RT found in rodents (Reinagel, 2013b; Uchida \& Mainen, 2003). In contrast, accuracy across RT in nonhuman primates is reported to initially increase, then progressively decrease with longer RT (Reppert et al., 2018), which may reflect an increased urgency signal over time (Cisek et al., 2009; Thura et al., 2014).

In the experiment described here, accuracy was measured across RT for the discrimination of simple visual patterns. In order to examine the effects of RT on stimulus processing, comparisons were made across levels of stimulus strength and computational load. Analysis was not directed at speedaccuracy tradeoff, but instead focused on the RT-accuracy profile in an unconstrained task. Rats initiated trials with a free operant procedure, and they were allowed unlimited time to examine stimuli before responding. Three factors were varied: (1) stimulus contrast (2) stimulus duration, and (3) computational load, where discrimination was based upon either solid lines (low load) or dot grids that required perceptual grouping (high load). Manipulation of computational load was performed in order to examine effects of increased demands on stimulus processing. For experiments described here, computational load refers to neural processing of the stimulus, and not to cognitive factors such as working memory, or to factors related to stimulus magnitude, such as luminance contrast or duration. Instead, computational load refers to the process of perceptual grouping, which develops progressively following stimulus presentation (Beck \& Palmer, 2002; Kimchi, 2000; Kurylo, 1997; Kurylo \& Bukhari, 2017; Palmer et al., 2003; Razpurker-Apfeld \& Kimchi, 2007). In the process of perceptual grouping, neural algorithms serve to integrate stimulus elements into coherent forms (Roelfsema, 2006). Neural mechanisms proposed to mediate grouping include increased response among grouped contours (Roelfsema et al., 2004) or among neurons encoding common features (Roelfsema \& Houtkamp, 2011), enhanced connection strength (Roelfsema \& Houtkamp, 2011), or synchronous activity among grouped components (Nicolaev et al., 2010). In each case, the primary neural representation derived from the afferent signal undergoes additional processing (i.e., increased computational load) to generate a response pattern that enables discrimination.

It was hypothesized that increased RT reflects additional processing, and therefore accuracy should progressively rise with longer RT. As such, a progressive decrease of stimulus contrast, a decrease in presentation time, or an increase in computational load should reduce accuracy gain with longer RT. This hypothesis is consistent with literature on speed-accuracy tradeoff. If increased RT reflects 
perceptual choice factors, such as delayed commitment to a response due to uncertainty, then accuracy should progressively decline with longer RT. With the alternative mechanism, increasing demands on processing should produce an increased rate of decline across RT. As such, decreased accuracy with longer RT is inconsistent with literature on speed-accuracy tradeoff, in terms of accumulating information over time.

\section{Methods}

\section{Subjects}

Eleven female Long-Evans hooded rats served as subjects. Animals were water restricted and received water as reward during sessions, as well as ad lib for one hour following sessions. Sessions occurred on four or five days each week, and animals were allowed water ad lib on remaining days. This study was conducted in accordance with the National Institutes of Health Guide for the Care and Use of Laboratory Animals, and it was approved by the Institutional Animal Care and Use Committee of Brooklyn College.

\section{Apparatus}

Behavioral measurements were made in a customized operant conditioning chamber (Figure 1C). In order to specify stimulus parameters relative to the corneal surface, rats viewed stimuli from a fixed position. A metal funnel extended $3 \mathrm{~cm}$ outside of the chamber, and rats viewed stimuli through holes positioned over each eye. Placement of the rat's head completely within the funnel disrupted an infrared (IR) light beam connecting an emitter/detector pair mounted at the tip of the funnel.

A
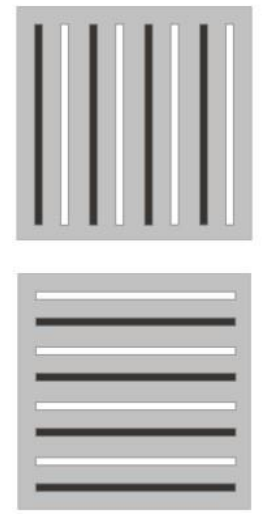

B
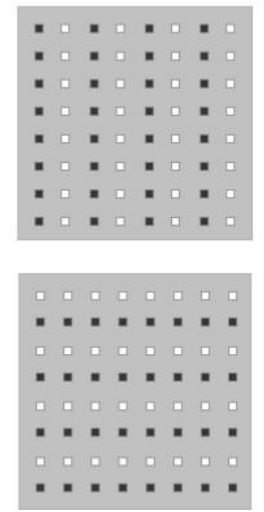

C

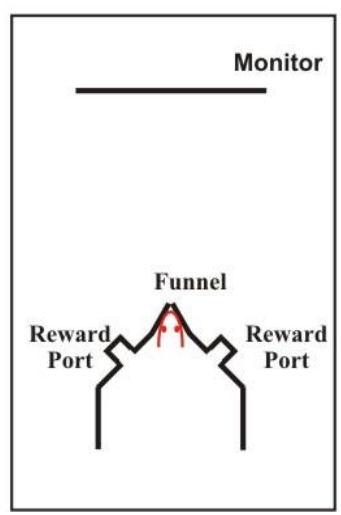

D

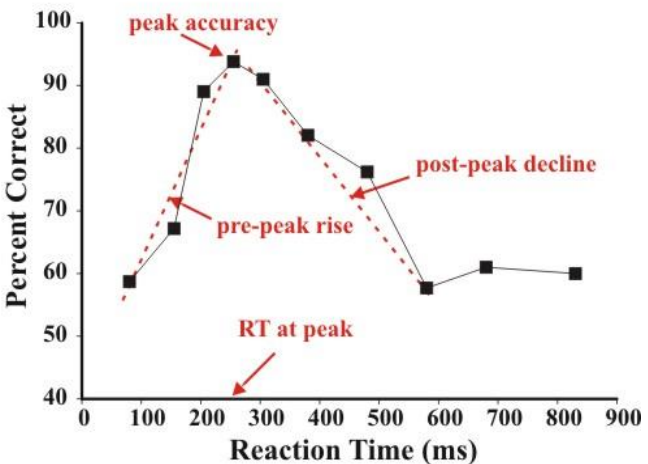

Figure 1. Vertical and horizontal stimuli for (A) solid line and (B) grouped dots conditions. C: Top of view of test chamber, indicating central funnel for viewing stimuli on monitor, with approximate position of rat's head, flanked by left and right reward wells associated with response choice. D: Example of data used for statistical analyses. Peak accuracy and RT at peak accuracy are based upon maximum percent correct at each RT bin. Dashed lines depict linear fit to data points preceding and including peak accuracy, and linear fit to data points including and following peak accuracy, extending to asymptotic level.

Rats had access to enclosed drinking ports positioned on each side of the funnel. Entering the enclosure for the drinking ports was monitored with IR pairs. A measured amount of water (approximately $0.04 \mathrm{ml}$ ) could be delivered to each port by means of a solenoid driven valve. Circuitry for the IR pairs and solenoids were interfaced via solid-state relay switches to a computer. Stimulus generation, data collection, and trial events were controlled by customized computer software (Bukhari \& Kurylo, 2008). 


\section{Stimuli}

In an otherwise darkened room, stimuli were presented on a computer monitor (Trinitron CPD 4401) controlled by a graphics adaptor (NVidia GeForce FX5200) set to 1280 x 1024 pixel resolution at $60 \mathrm{~Hz}$. Stimulus duration was linked to the monitor's vertical synchronization signal. Stimuli subtended a visual angle of $14.0^{\circ}$, and they consisted of either a set of parallel solid lines or a grid of dots (Figure 1A and $\mathrm{B})$.

Solid line stimuli. Solid-line stimuli consisted of 8 parallel lines, each $1.1^{\circ}$ x $25.9^{\circ}$, oriented either vertically or horizontally (Figure 1A). Lines alternated in luminance $\left(0.04\right.$ and $\left.38.2 \mathrm{~cd} / \mathrm{m}^{2}\right)$ on a gray background $\left(15.0 \mathrm{~cd} / \mathrm{m}^{2}\right)$.

Dot grid stimuli. Dot grids consisted of an $8 \times 8$ array of squares, equally spaced by $3.1^{\circ}$ vertically and horizontally (Figure 1B). As such, elements superimposed the location used with solid-line stimuli. Luminance of elements and background match that of solid-line stimuli. Elements with the same luminance were organized along either the vertical or horizontal orientation. Dot grids could thereby be perceptually organized as a series of lines by means of grouping by luminance similarity.

\section{General Procedure}

Rats discriminated vertical and horizontal patterns. Rats initiated trials by placing their head into the funnel. Rats viewed stimuli at a distance of $24 \mathrm{~cm}$ and elevated by $10^{\circ}$, centered within the binocular region of the visual field. Following a randomized delay, which ranged from $500-1500 \mathrm{~ms}$, a stimulus appeared, and rats indicated whether the pattern appeared to be vertical or horizontal by entering either the left or right drinking port (2-alternative forced-choice (2AFC) procedure). Reward was delivered immediately upon rats entering the correct drinking port. The association of port location and stimulus orientation was counterbalanced across rats. Rats could then immediately initiate a new trial. Daily sessions occurred for approximately one hour, in which rats completed approximately 250 trials.

Some procedures employed here differ from previous research employing psychophysical techniques in rodent (Abraham et al., 2004; Reinagel 2013a, b; Rinberg et al., 2006; Uchida \& Mainen, 2003). Notably, procedures used here did not employ an experimenter-controlled trial initiation, imposed inter-trial interval or a time-out penalty for errors. Instead, animals regulated trial initiation and pace, which increased opportunity for trial initiation. In addition, 2AFC was not based upon identifying a target among multiple stimuli presented simultaneously, or a go/not-go response identifying stimulus presence. Instead, different, parallel response choices were associated with each stimulus types, presented individually on each trial. In order to monitor stimulus control of behavioral responses, stimuli were presented at a randomized delay from trial initiation, and RT analysis was time-locked to stimulus onset, and not trial initiation. In this way, rats are unable to anticipate stimulus onset and respond after a fixed delay. Finally, viewing stimuli from within a fixed location ensured stimulus specificity relative to the corneal surface (Dean, 1981, 1990).

\section{Training}

Following magazine training to become familiar with the chamber and reward delivery, rats were trained to nose-poke the central funnel, and then to remain stationary within the funnel for increasing lengths of time, culminating with a $500 \mathrm{~ms}$ hold period. Rats were then trained to respond to the onset of a stimulus by removing their head from the funnel and entering a drinking port. Finally, rats were trained to discriminate solid line patterns by responding to the correct drinking port. Across training sessions, stimulus onset times progressively increased to a range of $500-1500 \mathrm{~ms}$, and RT to stimulus onset was progressively restricted to 80 - $400 \mathrm{~ms}$. Increased range of stimulus onset and decreased RT window was contingent upon rats' performance, requiring $90 \%$ correct responses across a session. Specifically, the RT window was narrowed by $20 \mathrm{~ms}$ after reaching the performance criterion. 
Following discrimination training with solid lines, stimuli were broken into closely spaced sequences of square elements where proximity served as a cue for grouping. Spacing along the grouped orientation was progressively increased across sessions, contingent upon performance. With equally spaced elements, grouping relied entirely upon luminance similarity in order to discriminate dot patterns. Stimuli used for training were set to a contrast of $93 \%$ with no restriction on duration.

\section{Testing}

Once animals reached $90 \%$ correct discrimination accuracy, testing sessions were begun. RT was measured as the time from stimulus onset to head withdrawal from the funnel. RT between 30 and $929 \mathrm{~ms}$ were used for analysis, which is similar to RT used for human (Plainis \& Murray, 2000) and rodent (Histed, Carvalho, \& Maunsell, 2012) studies. For testing sessions, reward was not contingent upon RT (as used for training), and reward was delivered for all correct discriminations.

Data for the contrast and duration conditions were collected in separate, interleaved sessions. The contrast conditions contained six stimulus conditions (three contrast and two computational loads). The duration condition contained eight stimulus conditions (four durations and two computational loads). Because of the relatively large number of stimulus conditions used, in each experimental session, three of all possible stimulus conditions were tested, selected randomly without replacement. Within a session, stimulus conditions were interleaved. Performance on each stimulus condition was based upon approximately 175 trials, collected across multiple sessions. Data were typically collected on two days per week, with training on alternate days. Parameters used during the interspersed training sessions were the same as those used in initial training. Data acquisition required approximately five sessions for the contrast conditions, and seven sessions for the duration condition.

Condition 1: Stimulus Contrast. For the contrast condition, discrimination accuracy across RT was recorded for three levels of stimulus contrast: 11, 31, and 92\% (Michelson). In each case, stimuli remained on until rats withdrew their head from the funnel (i.e., continuous duration).

Condition 2: Stimulus Duration. For the duration condition, measurements were made for four levels of stimulus duration: continuous (i.e., stimuli remained on until rats withdrew from the funnel), 100, 50, and $25 \mathrm{~ms}$. In each case, stimulus contrast was set to $92 \%$.

\section{Analyses}

Data were collapsed within bins of RT. For analysis of RT frequency distributions, all bins were $100 \mathrm{~ms}$. For accuracy distributions, bins were $50 \mathrm{~ms}$ for RT between 130-329 ms, and $100 \mathrm{~ms}$ for all other RTs. In all cases, response frequency or accuracy were compared using the Friedman test. Analyses were made of peak performance, as well as slopes describing change in accuracy leading up to, and declining after, peak performance, each inclusive of peak accuracy. Criteria for data assignment to rising and declining slopes was established a priori and applied equally to all animals in all conditions. Specifically, rising slopes were based upon data from the shortest RT through to the first RT with peak performance. This first highest value was assigned to the peak level. Declining slopes were based upon data from the peak level to the lowest level that occurred with increased RT. For slopes that declined to an asymptote, the first occurrence of the lowest level was used for declining slope calculation. A representative example of performance across RT, with the derived values used in analysis, is shown in Figure 1D. It can be seen that calculating the declining slope to the asymptote better represented the slope of decreased accuracy than would performance at the longest RT recorded.

\section{Statistical Analysis}

Comparisons across levels of contrast or levels of duration were performed with the Friedman test, which is a non-parametric analysis of variance. Friedman test statistic is reported as $\chi_{r}^{2}$. For cases of significant effects, post-hoc tests were applied to determine which pairs of conditions differed 
significantly. Post-hoc tests were performed with the Wilcoxon signed-rank test, with Bonferroni correction for multiple comparisons. In addition, comparisons of the solid lines vs. dots conditions were performed at each level of contrast or duration by means of the Wilcoxon test, also with Bonferroni correction.

\section{Results}

Performance was stable across data collection sessions, where percent correct varied by no more than $6 \%$ for each stimulus condition. In a few cases early in the period of data collection (representing fewer than $2 \%$ of sessions), performance was inconsistent with other sessions, and data were not included in statistical analyses. Performance in these cases stabilized in subsequent sessions.

\section{RT Frequency Distributions}

An examination was first made of the frequency distributions across RT. Percent responses as a function of RT is shown in Figures 2A and 2B (for the contrast condition) and in Figures 3A and 3B (for the duration condition). Across all conditions, response frequency peaked between approximately 130 $329 \mathrm{~ms}$, followed by a progressive decline, where few responses occurred at the longest RT. Comparisons were made of the percent responses at the peak response rate, as well as the RT at which peak responses occurred.

\section{Stimulus Contrast}

Peak frequency. Response frequency distributions are shown in Figure 2A and 2B. As contrast was reduced, peak response frequency declined. For solid lines, peak response frequency differed significantly with different contrast levels (Friedman analysis of variance: $\chi^{2} r(2)=14.6, p=.001$ ). Post hoc analysis of paired contrasts indicated significant differences between high and low contrast (Wilcoxon signed-rank test: $\mathrm{Z}=-2.71, p=.007$ ) and between medium and low contrast (Wilcoxon $\mathrm{Z}=$ 2.81, $p=.005$ ), while the difference between high and medium contrast (Wilcoxon, $p=.028)^{1}$ did not reach criterion for multiple-comparison correction (Bonferroni-adjusted significance $=.017$ ). Similar results were found for dots, where a significant decline in peak responses occurred (Friedman $\chi^{2} r(2)=$ $12.0, p=.002)$. Post-hoc comparisons of contrasts indicated significant differences between all paired contrast levels (Wilcoxon $\mathrm{Z}=-2.37, p=.018$ for all pairs). Comparing solid lines and dots conditions, peak frequency (collapsed across contrast levels) did not differ significantly (Wilcoxon, $p=.747$ ).

RT at peak frequency. The RT at which peak responses occurred did not differ significantly across contrast levels for solid lines (Friedman, $p=.714$ ) or dots (Friedman, $p=.417$ ). Solid line and dots conditions did not differ significantly (Wilcoxon, $p=.739$ ).

\section{Stimulus Duration}

Peak frequency. Frequency distributions were similar across stimulus durations (Figure 3A and 3B). For all durations, peak response frequency occurred at $130-229 \mathrm{~ms}$, and declined similarly at longer RT. For solid lines, response frequencies were not identical (Friedman $\chi^{2} r(3)=8.36, p=.039$ ), however there was no clear effect of stimulus duration. Paired comparisons of stimulus duration did not reach criterion for multiple-comparison correction (Bonferroni-adjusted significance $=.008$ ). For grouped dots, response frequency declined significantly across duration (Friedman: $\chi^{2} r(3)=10.02, p=.018$ ), although no paired durations differed significantly with Bonferroni correction. A significant difference existed in

\footnotetext{
${ }^{1}$ In all cases where statistical analyses produced non-significant results, only the $p$ values are reported for ease of reading. Test values can be provided upon request to the authors.
} 
percent responses at peak frequencies between the solid lines $(M=33.7 \%, S D=7.0)$ and dots $(M=$ $42.5 \%, S D=9.4)($ Wilcoxon $Z=2.78, p=.005)$.

RT at Peak Frequency. RT at peak did not differ significantly across duration (Friedman, solid lines: $p>.640$; dots: $p=.356)$. RT at peak frequency did not differ significantly between the solid lines and dots conditions (Wilcoxon, $p=.058$ ).

\section{Stimulus Contrast Condition}
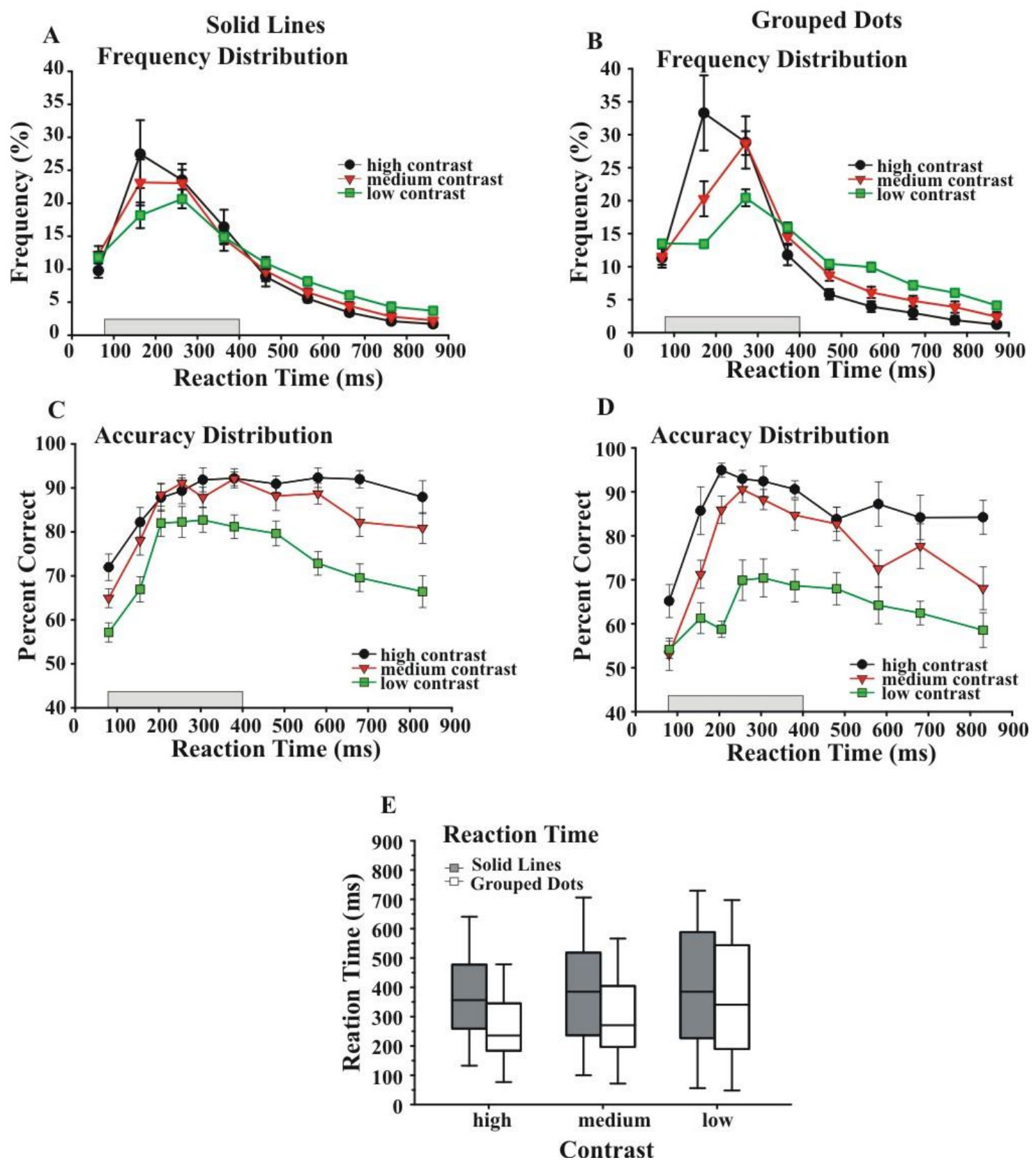

Figure 2. Performance with solid-line and grouped-dot stimuli across levels of stimulus contrast. RT are relative to stimulus onset. Each data point for A-D is based upon 10 (for solid lines) or 8 rats (for grouped dots), each of which was collected across approximately 175 trials. A and B: Response frequency distributions; C and D: Percent correct for pattern discrimination across bins of RT. Error bars represent SEM. Shaded region depicts period during which reward could be delivered during training. E: Reaction time across contrast for solid-line and grouped-dot stimuli. Center lines depict median, box borders depict $25^{\text {th }}$ and $75^{\text {th }}$ percentiles, error bars depict $10^{\text {th }}$ and $90^{\text {th }}$ percentiles. 


\section{Stimulus Duration Condition}
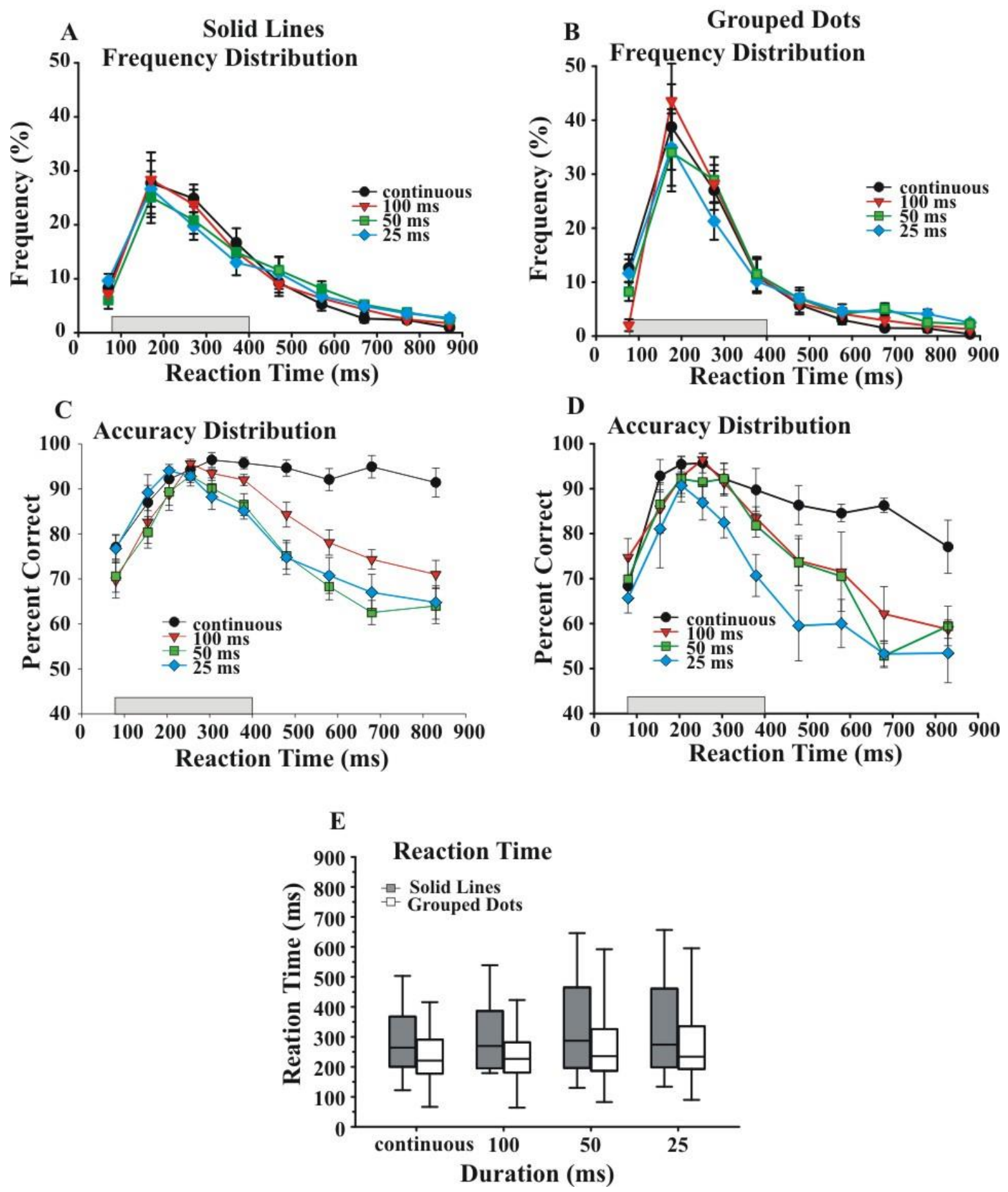

Figure 3. Performance with solid-line and grouped-dot stimuli across levels of stimulus duration. RT are relative to stimulus onset, and each data point for A-D represents the mean performance across rats. A and B: Response frequency distributions; C and D: Percent correct for pattern discrimination across bins of RT. Error bars represent SEM. Shaded region depicts period during which reward could be delivered during training. E: Reaction time across duration for solid-line and grouped-dot stimuli. Center lines depict median, box borders depict $25^{\text {th }}$ and $75^{\text {th }}$ percentiles, error bars depict $10^{\text {th }}$ and $90^{\text {th }}$ percentiles. 


\section{Accuracy Distributions}

Discrimination accuracy was determined for each RT bin. In order to better describe accuracy patterns during the period of greatest responding, analyses were made for $50 \mathrm{~ms}$ bins for RT between 130 - 329 ms. Mean accuracy as a function of RT is depicted in Figures 2C, 2D (for the contrast condition) and in Figures 3C and 3D (for the duration condition). In addition, mean RTs for the solid lines vs. dots stimuli are shown in Figures $2 \mathrm{E}$ (for contrast) and $3 \mathrm{E}$ (for duration).

Analyses were made of RT to peak accuracy, the pre-peak slope of rise in performance, and the post-peak slope of decline in performance. Analysis of slope targets gain in performance as a function of RT, which represents the rate at which RT affects performance accuracy. Slope metrics do not contain absolute levels of accuracy. Analysis of absolute accuracy is instead based upon mean accuracy at peak performance. Slopes were based upon the linear regression of accuracy as a function of RT, either before peak (from $30 \mathrm{~ms}$ to peak accuracy) and after peak (peak accuracy to $929 \mathrm{~ms}$, or to the asymptotic level near chance performance). Subject means depicted in Figures 2C and 2D reflected performance by individual rats. A representative example of individual performance is show in Figure 4, where performance rose to a peak, then progressive decline across longer RT. All rats tested were found to follow the trends found in mean performance (Figures 2 and 3).
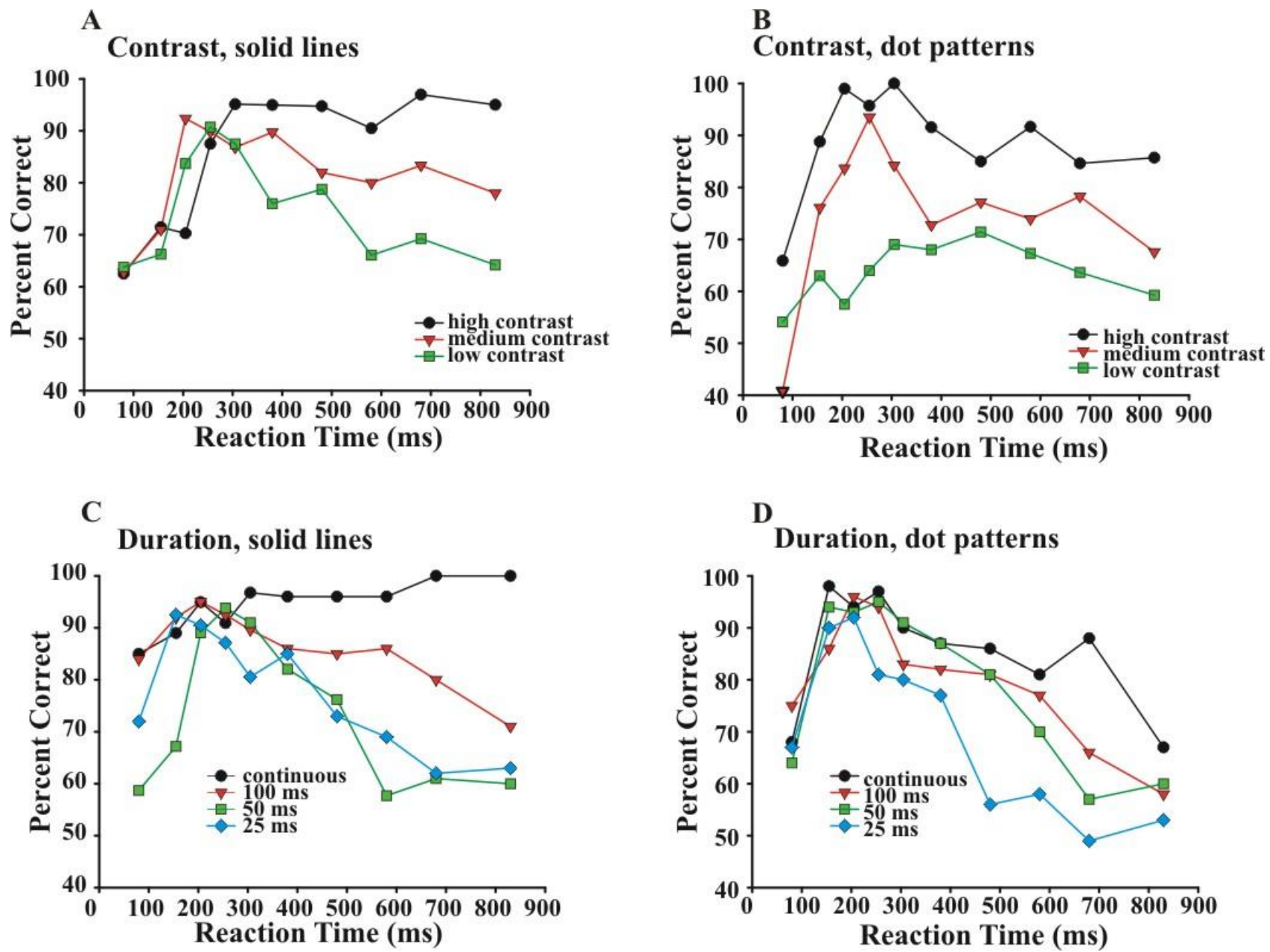

Figure 4. Example of performance by an individual rat for each stimulus condition: A. contrast with solid lines, B. contrast with grouped dots, $\mathbf{C}$. duration with solid lines, and $\mathbf{D}$. duration with grouped dots. 


\section{Stimulus Contrast}

Contrary to the hypothesis that longer RTs would produce a progressive increase in accuracy, with increased RTs, accuracy improved to a peak level, then progressively declined (Figure 2C and 2D). With high contrast stimuli, performance improved more quickly, and peak performance reached earlier, with grouped dots than with solid lines. In addition, reducing contrast had different effects for solid lines and dots conditions.

RT to peak accuracy. RT to peak accuracy as a function of contrast is shown in Figure 5A. For solid lines, reduced contrast did not significantly change RT to peak accuracy (Friedman two-way analysis of variance, $p=.905)$. For grouped dots, reduced contrast produced longer RT to peak accuracy (Friedman $\left.\chi^{2} r(2)=6.08, p=.046\right)$. Post-hoc analysis of paired contrast levels indicated a significant difference between the high and low levels (Wilcoxon $Z=2.43, p=.015$ ), whereas other paired levels did not differ significantly (low to medium: $p=.157$; medium to high: $p=.084$; Bonferroni-adjusted significance level: $p=.016$ ). Comparing solid-line and dots conditions at each contrast, dots produced a significantly longer RT to peak at low contrast (Wilcoxon $Z=-2.46, p=.014$; Bonferroni-adjusted significance: $p=.016)$, whereas performance did not differ significantly for high $(p=.236)$ and medium $(p=1.00)$ contrasts.
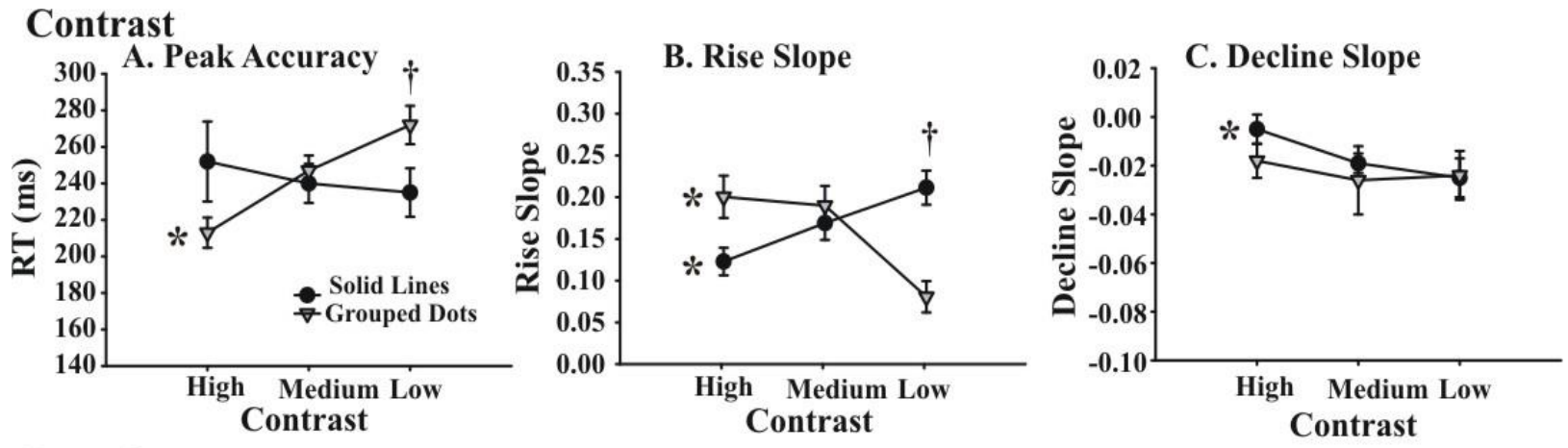

\section{Duration}
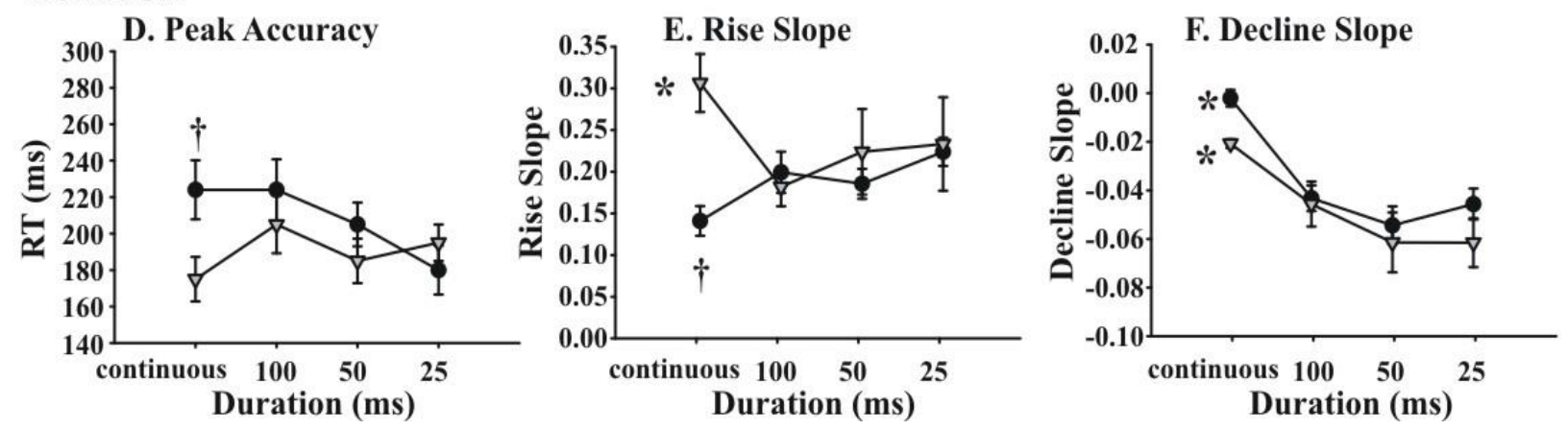

Figure 5. Characteristics of accuracy for the stimulus contrast (A - C) and stimulus duration (D - F) conditions. A and D: RT at peak accuracy; $\mathbf{B}$ and $\mathbf{E}$ : slope representing progressive increase in accuracy before peak; $\mathbf{C}$ and $\mathbf{F}$ : slope representing decline in accuracy following peak. Asterisks represent significant differences across levels of contrast or duration, and $\dagger$ represent significant differences between lines and dots conditions. Error bars represent SEM.

Pre-peak rise in accuracy. Pre-peak rise in accuracy is shown in Figure 5B). For solid lines, slope steepness increased significantly with reduced contrast (Friedman $\chi^{2} r(2)=6.20, p=.047$ ), although paired contrast levels did not differ significantly (high to medium: $p=.114$; high to low: $p=.047$, medium to low, $p=.169$; Bonferroni-adjusted significance: $p=.016$ ). For dots, slope steepness decreased significantly with reduced contrast (Friedman $\chi^{2} r(2)=9.00, p=.009$ ). Post-hoc analysis indicated 
significant differences between high and low contrast (Wilcoxon $Z=2.54, p=.012$ ) and between medium and low contrast (Wilcoxon $Z=2.55, p=.011$ ), whereas high and medium contrast did not differ significantly (Wilcoxon, $p=.767$ ). Comparing the solid line to dots conditions at each contrast, dots showed significantly lower slopes at low contrast (Wilcoxon $Z=2.55, p=.011$ ), whereas conditions did not differ significantly at high (Wilcoxon, $p=.066$ ) or medium contrasts (Wilcoxon, $p=.594$ ).

Post-peak decline in accuracy. Post-peak decline in accuracy is shown in Figure 5C. For solid lines, slope steepness increased significantly with reduced contrast (Friedman $\chi^{2} r(2)=7.85, p=.02$, indicating a faster decline in accuracy after reaching peak performance. Post hoc analysis indicated differences between high and medium contrast (Wilcoxon $Z=2.43, p=.015$ ), whereas high and low contrasts (Wilcoxon, $p=.047$ ) and medium and low contrasts (Wilcoxon, $p=.102$ ) did not differ significantly. For dots, slopes did not differ significantly across contrast (Friedman, $p=.417$ ). Comparing the solid line to the dots conditions, slopes did not differ significantly at any contrast (Wilcoxon, high contrast: $p=.293$; medium: $p=.600$; low: $p=.345$ ).

\section{Stimulus Duration}

Accuracy across RT for the duration condition was similar to that found for contrast, where accuracy rose to a peak, then declined with longer RT (Figure 3C and 3D). With limited stimulus duration, accuracy declined following stimulus offset. In addition, accuracy across RT differed between the solid lines and dots condition.

RT to peak accuracy. RT to peak accuracy is shown in Figure 5D. For solid lines as well as grouped dots, RT to peak accuracy did not differ significantly across duration (Friedman, solid lines: $p=$ .136; dots: $p=.494$ ). Comparing solid lines and dots at each level of duration, RT to peak accuracy was significantly shorter for dots with the continuous stimulus (Wilcoxon $Z=-2.60, p=.009$ ), whereas conditions did not differ significantly at other durations (Wilcoxon, $100 \mathrm{~ms}: p=.046 ; 50 \mathrm{~ms}: p=1.0 ; 25$ ms: $p=.655$; Bonferroni-adjusted significance level: $p=.013)$.

Pre-peak rise in accuracy. Pre-peak rise in accuracy is shown in Figure 5E. For solid lines, prepeak rise differed significantly across duration (Friedman $\chi^{2} r(3)=8.01, p=.046$ ), although paired duration levels did not differ significantly (Bonferroni-adjusted significance level: $p=.008$ ). For dots, pre-peak rise did not differ significantly across duration (Friedman, $p=.127$ ). Comparing solid lines and dots conditions, slope was significantly steeper for dots with the continuous stimulus (Wilcoxon $Z=2.52$, $p=.008)$, whereas conditions did not differ significantly at other durations $(100 \mathrm{~ms}: p=.068,50 \mathrm{~ms}, p=$ .893; 25 ms: $p=.686$; Bonferroni-adjusted significance: $p=.013$ ).

Post-peak decline in accuracy. Post-peak slopes are shown in Figure 5F. For solid lines, slope steepness increased significantly with shorter durations (Friedman $\chi^{2} r(2)=16.35, p=.001$ ). Post-hoc analysis indicated significant differences between continuous and $100 \mathrm{~ms}$ (Wilcoxon $Z=2.67, p=.008$ ) and between continuous and $50 \mathrm{~ms}$ (Wilcoxon $Z=2.67, p=.008$ ), whereas other paired durations did not differ significantly (Bonferroni-adjusted significance: $p=.008$ ). For dots, slopes also increased with reduced stimulus duration (Friedman $\chi^{2} r(2)=9.18, p=.026$ ). Post-hoc analysis indicated significant differences between the continuous and $25 \mathrm{~ms}$ durations (Wilcoxon $\mathrm{Z}=2.67, p=.008$ ), whereas other paired durations did not differ significantly. The solid lines and dots conditions did not differ significantly at any duration (Wilcoxon, continuous: $p=.021,100 \mathrm{~ms}: p=.953 ; 50 \mathrm{~ms}: p=.860 ; 25 \mathrm{~ms}: p=.213$; Bonferroni-adjusted significance level: $p=.013$ ).

\section{Discussion}

An examination of accuracy across RT was made for a simple visual discrimination task in rats. The level of accuracy across RT followed a peak function, where performance peaked at a mean of 218 ms across conditions, then either remained stable, or declined with longer RT. The rate of change in accuracy, and the RT at which accuracy peaked, interacted with stimulus contrast, stimulus duration, and 
processing load. Specifically, reduced contrast, which diminished the strength of stimulus representation, generally slowed the rise in accuracy, and reduced the overall accuracy level. Reduced duration, which limited availability of the afferent signal, also slowed the rise in accuracy, suggesting effects from the fading stimulus representation.

The early RT phase showed a progressive increase in accuracy. A similar pattern of response by rats was reported for odor discrimination (Uchida \& Mainen, 2003) and discrimination of coherent motion (Reinagel, 2013). Early responses appear to occur before completion of processing, thereby increasing error level. As with odor discrimination (Uchida \& Mainen, 2003), discrimination accuracy rose to an asymptotic level. This pattern may reflect a high level of confidence following the completion of processing.

Contrary to the hypothesis that accuracy would progressively increase with longer RT, accuracy at longer RT either remained constant, or declined, where additional processing time did not benefit accuracy. A similar response pattern was found with odor discrimination, where performance degraded beyond an RT of $250 \mathrm{~ms}$ for higher levels of difficulty (Uchida \& Mainen, 2003). Post-peak effects may reflect response delay resulting from factors associated with perceptual decision. With the exception of contrast with dot patterns, reducing stimulus contrast or duration accelerated decline in accuracy, and increased error rates with delayed responding. With lower stimulus strength, response delay is thereby associated with diminished performance. Unlike lower stimulus strength, with high stimulus strength, accuracy remained at nearly peak levels across longer RT. For trials with high accuracy and long RT, result suggests extended processing until a sufficient level certainty is achieved, although such validation requires high stimulus strength.

Reduced contrast broadened RT frequency distributions, decreased the rise in accuracy, and reduced overall performance. Increased RT with reduced stimulus quality occurs for simple (Marshall \& Brandt, 1980; Ulrich, Rinkenauer, \& Miller, 1998) and choice RT (Miller et al., 1999; Mulder et al., 2013), where change in accuracy may relate to change in accumulated sensory information (Mulder et al., 2013). Across levels of contrast, the RT-accuracy profiles for grouped dots paralleled changes in frequency distribution, reflecting similar dynamics for response rate and performance. However, for solid lines, although overall performance declined with reduced contrast, accuracy peaked earlier. The more progressive rise in accuracy with high contrast suggests benefits of increased processing, whereas for low contrast, accuracy reach a limit early, and did not further improve with additional processing.

With reduced duration of solid lines, the initial decline in accuracy was more consistently linked to stimulus offset (occurring $155 \mathrm{~ms}$ following offset of both the 25 and $50 \mathrm{~ms}$ stimuli) than to stimulus onset (180 and $205 \mathrm{~ms}$ for the 25 and $50 \mathrm{~ms}$ stimuli, respectively). A marked decline in accuracy did not occur with continuous presentation of the stimulus. Because accuracy declined more quickly with shorter stimulus duration, results suggest that fading of stimulus representations contribute to the decline in accuracy. Decline in accuracy with brief stimuli may reflect the absence of an afferent signal, where stimulus representations fade. Such an effect may represent reduction in the memory trace of the stimulus, which reduces the ability to discriminate patterns after the stimulus is removed. At a physiological level, the sensory response from the retinal-thalamic afferent pathway is received by visual area 1 (V1), which is the initial cortical region processing the visual signal. Following offset, the afferent signal continues to arrive in area V1 for approximately $40 \mathrm{~ms}$ (corresponding to stimulus response latencies found in $\mathrm{V} 1$ of rats (Tafazoli et al., 2017; Vermaercke et al., 2014). Following this period, it was found here that performance was stable or improved in the absence of stimulus input for approximately $105 \mathrm{~ms}$, after which accuracy began to decline. This $105 \mathrm{~ms}$ period may reflect factors such as neural persistence of the stimulus representation, intrinsic processing of pattern discrimination, or processing in cortical areas beyond V1. These results suggest that peak performance to discriminate line patterns requires approximately $225 \mathrm{~ms}$ (peak RT for continuous presentation), the last $105 \mathrm{~ms}$ of which is not reliant upon an afferent signal. Reinagel (2013b) examined the relationship between RT and discrimination of coherent motion direction. With limited stimulus duration (25 ms to $225 \mathrm{~ms})$, accuracy progressively decreased following stimulus offset. Consistent with results presented here, the RT at which performance 
began to decline appeared related to stimulus offset. Together, results suggest that stimulus processing continues in the absence of the stimulus.

Decline in performance with longer RT for the continuous presentation condition (Figure 3D) may reflect cognitive factors not directly related to stimulus processing. With longer RT, accuracy may be affected by factors such as reduced motivation or increased distraction. Direct measures of such cognitive processes are required to investigate their contribution to performance accuracy with increased RT.

In order to ensure behavioral control by the visual stimulus, reward delivery during training was restricted to responses that occurred within a window of 80 to $400 \mathrm{~ms}$ from stimulus onset. This procedure encouraged responding to stimulus events, and discouraged animals from simply remaining in the funnel for extended periods and receiving reward on $50 \%$ of trails, which may be sufficient to maintain behavioral without attending to the stimulus. The possibility exists that restriction during training may have encouraged quicker responses, where animals' expectation of reward opportunity declines after $400 \mathrm{~ms}$. As such, training procedures may have shifted response frequency distributions towards shorter RT (Figures 2A, 2B, 3A, and 3B). Whereas training procedures may have affected response frequency distributions, it is not clear whether the timescale or level of accuracy was affected. However, an interaction may have occurred between increased sampling of the stimulus signal versus decreased expectation of reward. The possibility exists that as response times approach or exceed $400 \mathrm{~ms}$, expectation of reward declines, where less care was given to the discrimination task. Possible effects of training constraints on RT-accuracy relationships may be further explored by measuring accuracy levels while systematically controlling response time.

Unexpectedly, the rise in accuracy occurred more quickly with dot patterns, which requires additional processing associated with perceptual grouping. This result may reflect the brief processing latencies for grouping by luminance. Specifically, the neural representation of luminance, neural mechanisms for grouping, and the formation of perceived grouping, occur at brief time scales. In rats, area V1 contains short latency neurons sensitive to luminance contrast (Tafazoli et al., 2017). Mechanisms for grouping by luminance are proposed to occur quickly through initial feedforward processing (Roelfsema, 2006; Roelfsema \& Houtkamp, 2011). At a perceptual level, grouping by luminance occurs with brief presentation of stimuli, where grouped patterns are formed with durations as short as $35 \mathrm{~ms}$ (Kurylo \& Bukhari, 2017). Discrimination of grouped patterns may be supported by the fast integration of luminance coding across levels of processing, reflected here at a behavioral level in RT-accuracy profiles.

\section{Summary}

For an unconstrained visual discrimination task in rats, results found here are characterized by two phases of response section. During an early phase, increased RT is accompanied by increased response accuracy, consistent with an accumulation of stimulus information. During a later phase, a decrease in accuracy occurs with further increase in RT, consistent with a change in response criteria and increased urgency signal. Together, the RT-accuracy profile followed a peak function, where increase and decline in accuracy varied with stimulus characteristics. Early responses, which occurred less often and with reduced accuracy, may reflect impulsive behavior that occurred before processing was complete. Across conditions, accuracy peaked at a mean of $218 \mathrm{~ms}$, followed by a period of maximal response rate. For longer RT, high accuracy was maintained with more salient stimuli, but progressively declined with reduced stimulus contrast or duration. With lower stimulus strength, higher computational load slowed the rise, and accelerated the decline, in accuracy. With high stimulus strength, higher computational load increased the rise in accuracy. This result may reflect characteristics of grouped patterns that facilitate discrimination, such as the integration of stimulus elements that occurs early in processing. Together, these results suggest two phases of the RT-accuracy profile that occur before and after optimal performance, each of which differ in the relative contribution of stimulus processing and decision certainty. 


\section{Acknowledgements}

Funding: This work was supported by the City University of New York PSC-CUNY Research Award Program (award \# 69491-00 47) and the National Science Foundation (Award \# 1461099).

\section{Conflict of Interest}

No conflict of interest exists for any of the authors.

\section{References}

Abraham, N. M, Spors, H., Carleton, A., Margrie, T. W., Kuner, T. \& Schaefer, A. T. (2004). Maintaining accuracy at the expense of speed: Stimulus similarity defines odor discrimination time in mice. Neuron, 44, 865-876.

Beck, D. M., \& Palmer, S. E. (2002). Top-down influence on perceptual grouping. Journal of Experimental Psychology: Human Perception and Performance, 28, 1071-1084.

Bukhari, F., \& Kurylo, D. D. (2008). Computer programming for generating visual stimuli. Behavior Research Methods, 40, 38-45.

Carandini, M., \& Churchland, A. K. (2013). Probing perceptual decisions in rodents. Nature Neuroscience, 16, 824831.

Cassey, P., Heathcote, A., \& Brown, S. D. (2014). Brain and behavior in decision making. PLOS Computational Biology, 10, e1003700.

Cisek, P., Puskas, G. A., \& El-Murr, S. (2009). Decisions in changing conditions: The urgency-gating model. Journal of Neuroscience, 29, 11560-11571.

Dean, P. (1981). Visual pathways and acuity in hooded rats. Behavioral Brain Research, 3, 239-271.

Dean, P. (1990). Sensory cortex: Visual perceptual functions. In B. Kolb \& R. C. Tees (Eds), The cerebral cortex of the rat (pp. 275-307). Cambridge, MA: MIT Press.

Gavornik, J. P., \& Bear, M. F. (2014). Higher brain functions served by the lowly rodent primary visual cortex. Learning and Memory, 21, 527-533.

Hare, T. A., Schultz, W., Camerer, C. F., O'Doherty, J. P., \& Rangel, A. (2011). Transformation of stimulus value signals into motor commands during simple choice. Proceedings of the National Academy of Sciences, 108, $18120-18125$.

Heitz, R. P. (2014). The speed-accuracy tradeoff: History, physiology, methodology, and behavior. Frontiers in Neuroscience, 8, 1-19.

Heitz, R., \& Schall, J. D. (2012). Neural mechanisms of speed-accuracy tradeoff. Neuron, 76, 616-628.

Heitz, R., \& Schall, J. D. (2013). Neural chronometry and coherency across speed- accuracy demands reveal lack of homomorphism between computational and neural mechanisms of evidence accumulation. Philosophical Transactions of the Royal Society of London B, 368, 20130071

Histed, M. H., Carvalho, L. A., \& Maunsell, J. H. R. (2012). Psychophysical measurement of contrast sensitivity in the behaving mouse. Journal of Neurophysiology, 107, 758-765.

Kepecs, A., Uchida, N., Zariwala, H. A., \& Mainen, Z. F. (2008). Neural correlates, computation and behavioral impact of decision confidence. Nature, 455, 227-233.

Kiani, R., \& Shadlen, M. N. (2009). Representation of confidence associated with a decision by neurons in the parietal cortex. Science, 324, 759-764.

Kimchi, R. (2000). The perceptual organization of visual objects: A microgenetic analysis. Vision Research, 40, $1333-1347$.

Kurylo, D. D. (1997). Time course of perceptual grouping. Perception and Psychophysics, 59, 142-147.

Kurylo, D. D., \& Bukhari, F. (2017). Effects of pattern masks on the formation of perceptual grouping. Vision Research, 138, 29-39.

Kurylo, D. D., Chung, C., Yeturo, S., Lanza, J., Gorskaya, A., \& Bukhari, F. (2015). Effects of contrast, spatial frequency, and stimulus duration on reaction time in tats. Vision Research, 106, 20-26.

Marshall, L., \& Brandt, J. F. (1980). The relationship between loudness and reaction time in normal hearing listeners. Acta Otolaryngol, 90, 244-249.

Miller, J., Franz V., \& Ulrich, R. (1999). Effects of auditory stimulus intensity on response force in simple, go/nogo, and choice RT tasks. Perception and Psychophysics, 61, 107-119. 
Mulder, M. J., Keuken, M., C., van Maanen, V., Boekel, W., Forstmann, B., U., \& Wagenmaker, E.-J. (2013). The speed and accuracy of perceptual decisions in a random-tone pitch task. Attention, Perception, and Psychophysics, 75, 1048-1058.

Palmer, S. E., Brooks, J. L., \& Nelson, R. (2003). When does grouping happen? Acta Psychologica, 114, 311-330.

Palmer, J., Huk, A. C., \& Shadlen, M. N. (2005). The effect of stimulus strength on the speed and accuracy of a perceptual decision. Journal of Vision, 5, 376-404.

Plainis, S., \& Murray, I. J. (2000). Neurophysiological interpretation of human visual reaction times: Effect of contrast, spatial frequency and luminance. Neuropsychologia, 38, 1555-1564.

Ratcliff, R., \& Rouder, J. N. (1998). Modeling response times for two-choice decisions. Psychological Science, 9, 347-356.

Razpurker-Apfeld, I., \& Kimchi, R. (2007). The time course of perceptual grouping: The role of segmentation and shape formation. Perception and Psychophysics, 69, 732-743.

Reinagel, P. (2013a). Speed and accuracy of visual image discrimination by rats. Frontier in Neural Circuits, 7, 110.

Reinagel, P. (2013b). Speed and accuracy of visual motion discrimination by rats. PlosOne, 8, 1-8. Reppert, T. R., Servant, M., Heitz, R. P., \& Schall, J. D. (2018). Neural mechanisms of speed accuracy tradeoff of visual search: Saccade vigor, the origin of targeting errors, and comparison of the superior colliculus and frontal eye field. Journal of Neurophysiology, 120, 372-384.

Rinberg, D., Koulakov, A., \& Gelperin, A. (2006). Speed-accuracy tradeoff in olfaction. Neuron, 51, $351-358$.

Roelfsema, P. R. (2006). Cortical algorithms for perceptual grouping. Annual Review of Neuroscience, 29, $203-207$.

Roelfsema, P. R., \& Houtkamp, R. (2011). Incremental grouping of image elements in vision. Attention, Perception, and Psychophysics, 73, 2542-2572.

Roelfsema, P. R., Lamme, V. A. F., \& Spekreijse, H. (2004). Synchrony and covariation of firing rates in the primary visual cortex during contour grouping. Nature Neuroscience, 7, 982-991.

Roitman, J. D., \& Shadlen, M. N. (2002). Response of neurons in the lateral intraparietal area during a combined visual discrimination reaction time task. Journal of Neuroscience, 22, 9475-9489.

Schall, J. D. (2001). Neural basis of deciding, choosing, and acting. Nature Reviews, 2, 33-42.

Sigman, M., Jobert, A., LeBihan, D., \& Dehaene, S. (2007). Parsing a sequence of brain activations at psychological times using fMRI. NeuroImage, 35, 655-668.

Soma, S., Suematsu, N., \& Shimegi, S. (2014). Efficient training protocol for rapid learning of the two-alternative forced-choice visual stimulus detection task. Physiological Reports, 2, e12060.

Sternberg, S. (2011). Modula processes in mind and brain. Cognitive Neuropsychology, 28, 156-208.

Tafazoli, S., Di Filippo, A., \& Zoccolan, D. (2012). Transformation-tolerant object recognition in rats revealed by visual priming. Journal of Neuroscience, 32, 21-34.

Tafazoli, S., Safaai, H., Franceschi, G. D., Rosselli, F. B., Vanzella, W., Riggi, M., Buffolo, F., Panzeri, S., \&Zoccolan, D. (2017). Emergence of transformation-tolerant representations of visual objects in rat lateral extrastriate cortex. eLife, 6, e22794.

Thura, D., Cos, I., Trung, J., \& Cisek, P. (2014). Context-dependent urgency influences speed-accuracy trade-offs in decision-making and movement execution. Journal of Neuroscience, 34, 16442-16454.

Uchida, N., \& Mainen, Z. F. (2003). Speed and accuracy of olfactory discrimination in the rat. Nature Neuroscience, 6, 1224-1229.

Ulrich, R., Rinkenauer, G., \& Miller, J. (1998). Effects of stimulus duration and intensity on simple reaction time. Journal of Experimental Psychology: Human Perception and Performance. 24, 115-128.

Vermaercke, B., Gerich, F. J., Ytebrouck, E., Arckens, L., Op de Beeck, H. P., \& Van den Bergh, G. (2014). Functional specialization in rat occipital and temporal visual cortex. Journal of Neurophysiology, 112, 1963-1983.

Zoccolan, D. (2015). Invariant visual object recognition and shape processing in rats. Behavioral Brain Research, $285,10-33$. 